Check for updates

St George's, University of London, London, UK

2 Imperial College London, London, UK

Correspondence to: D Gill dipender.gill@imperial.ac.uk

Cite this as: BMJ 2021;374:n2136 http://dx.doi.org/10.1136/bmj.n2136 Published: 02 September 2021

\title{
Ronapreve for prophylaxis and treatment of covid-19
}

\section{Equitable allocation is a priority

\author{
David B Sidebottom, ${ }^{1}$ Dipender Gill²
}

On 20 August 2021, Ronapreve received conditional marketing authorisation for the prevention and treatment of covid-19 in the UK. ${ }^{1}$ Ronapreve (REGEN-COV in the US) comprises two monoclonal antibodies, casirivimab and imdevimab, that target the SARS-CoV-2 spike protein to reduce the risk and severity of covid-19 in selected patients. ${ }^{2-5}$ Although Ronapreve's approval represents a welcome expansion in the armamentarium against covid-19, it also brings difficult questions about who should be eligible for treatment.

The conditional marketing authorisation was based on two pivotal trials. ${ }^{2-4}$ The first randomised 4567 outpatients with covid-19 to placebo or intravenous Ronapreve. ${ }^{23}$ Eligible patients had at least one risk factor for severe disease, symptom onset within 7 days, and a positive SARS-CoV-2 reverse transcription polymerase chain reaction (RT-PCR) result within 72 hours. One-off treatment with $1200 \mathrm{mg}$ or $2400 \mathrm{mg}$ Ronapreve reduced the composite primary outcome of hospital admission or death by around $70 \%(1 \% \mathrm{v}$ $3.2 \%$ of controls, $\mathrm{P}=0.0024$ for $1200 \mathrm{mg} ; 1.3 \%$ v $4.6 \%$, $\mathrm{P}<0.0001$ for $2400 \mathrm{mg}$ ) and the median time to symptom resolution from 14 to 10 days.

The second trial evaluated prophylactic subcutaneous administration of $1200 \mathrm{mg}$ Ronapreve versus placebo in people without antibodies to covid-19 (seronegative) who had been exposed to SARS-CoV-2 by someone in their household. ${ }^{4}$ Those who initially had a negative PCR result were less likely to develop disease when administered Ronapreve within 96 hours of contact (relative risk reduction $66 \%$, $\mathrm{P}<0.001$ ), while any consequent symptomatic infections also resolved faster (1.2 weeks versus 3.2 weeks). In asymptomatic individuals with an initial positive PCR result, treatment with Ronapreve reduced the development of symptomatic disease by $31 \%(\mathrm{P}<0.038){ }^{1}$

The UK based Randomised Evaluation of Covid-19 Therapy (Recovery) trial also investigated Ronapreve, randomising 9785 hospital patients with covid-19 to usual care or usual care with $8000 \mathrm{mg}$ intravenous Ronapreve administered once only. 5 Time from admission to treatment ranged between 1 and 4 days, and a prespecified subgroup analysis found Ronapreve reduced mortality among patients without detectable antibodies (rate ratio $0.80,95 \%$ confidence interval 0.70 to 0.91 ) but not seropositive patients (1.09, 0.95 to 1.26$)$.

The trial data collectively support the use of Ronapreve for post-exposure prophylaxis and treatment of covid-19 in seronegative people, in both hospital and outpatient settings. However, given the variation in efficacy between seronegative and seropositive patients, antibody testing will be paramount for optimising its allocation. Care is needed to avoid repeating previous mistakes regarding assay selection. ${ }^{6}$ Although the trials varied in their choice of antibody tests, ${ }^{245}$ widely available commercial assays may be able to achieve sensitivity and specificity of at least $98 \% .^{7}$

\section{Patient selection}

NHS resources are finite, and the limited supply of Ronapreve will need to be allocated to those who stand to gain most benefit. This is particularly important given that indiscriminate use risks driving SARS-CoV-2 escape mutations. ${ }^{8}$ Despite high levels of vaccine uptake and seropositivity in the UK, ${ }^{9}$ some people are still susceptible to severe disease. Older people and those with multimorbidity mount weaker antibody responses to vaccination. ${ }^{10}$

Immunosuppressed people are at particularly high risk. ${ }^{11}{ }^{12}$ Furthermore, vaccine efficacy is expected to wane over time. ${ }^{13}$ Such factors will need to be coupled with antibody status when prioritising the most vulnerable patients for treatment. In the community, fast, coordinated pathways will have to deliver antibody testing and administer Ronapreve soon after viral exposure or disease onset. ${ }^{1}$

The disproportionate effect of covid-19 on poorer families, older people, and ethnic minorities is well documented. ${ }^{14}$ National coordination will be essential to facilitate equitable access to Ronapreve and minimise the unwarranted variation in care that has become commonplace in the NHS. ${ }^{15}$ The National Institute for Health and Care Excellence must step up once again to guide frontline clinicians in their decision making processes. ${ }^{16}$ Guidance should balance resource availability against anticipated benefits in distinct population subgroups. Living guidelines that are constantly reviewed and refined must take centre stage to ensure that practice matches emerging evidence. ${ }^{17}$

The conditional marketing authorisation of Ronapreve is an important development in the prevention and treatment of covid-19 but also creates notable challenges, including a requirement for scalable antibody testing, appropriate patient selection, and time critical treatment delivery. Clinical pathways established for Ronapreve through iterative development may also be transferable across emerging SARS-CoV-2 antibody therapies. ${ }^{18}$ Just as practice for other aspects of managing covid-19 have developed through the course of the pandemic, ${ }^{19}$ the approach for allocation of Ronapreve will have to be continually refined.

Competing interests: We have read and understood BMJ policy on declaration of interests and declare the following interests: DG is employed part-time by Novo Nordisk and has received consultancy fees from Policy Wisdom, outside of and unrelated to the submitted work. 
1 Medicines and Healthcare Products Regulatory Agency. Summary of product characteristics for Ronapreve. 2021. https://www.gov.uk/government/publications/regulatory-approval-of-ronapreve/summary-of-product-characteristics-for-ronapreve

2 Weinreich DM, Sivapalasingam S, Norton T, etalTrial Investigators. REGN-COV2, a neutralizing antibody cocktail, in outpatients with covid-19. N Engl J Med 2021;384:238-51. doi: 10.1056/NEJMoa2035002. pmid: 33332778

3 Weinreich DM, Sivapalasingam S, Norton T, etal. REGEN-COV antibody cocktail clinical outcomes study in covid-19 outpatients. [Preprint.] medRxiv 2021:2021.05.19.21257469. doi: 10.1101/2021.05.19.21257469

4 O'Brien MP, Forleo-Neto E, Musser BJ, etalCovid-19 Phase 3 Prevention Trial Team. Subcutaneous REGEN-COV antibody combination to prevent covid-19. N Engl J Med 2021. doi: 10.1056/NEJMoa2109682. pmid: 34347950

5 RECOVERY Collaborative Group. Casirivimab and imdevimab in patients admitted to hospital with COVID-19 (RECOVERY): a randomised, controlled, open-label, platform trial. [Preprint.] medRxiv 2021:2021.06.15.21258542. doi: 10.1101/2021.06.15.21258542

6 Gill D, Ponsford MJ. Testing for antibodies to SARS-CoV-2. BMJ2020;371:m4288. doi: 10.1136/bmj.m4288. pmid: 33315587

7 National SARS-CoV-2 Serology Assay Evaluation Group. Performance characteristics of five immunoassays for SARS-CoV-2: a head-to-head benchmark comparison. Lancet Infect Dis 2020;20:1390-400. doi: 10.1016/S1473-3099(20)30634-4

8 Starr TN, Greaney AJ, Addetia A, etal. Prospective mapping of viral mutations that escape antibodies used to treat COVID-19. Science 2021:371:850-4. doi: 10.1126/science.abf9302. pmid: 33495308

9 Ward H, Whitaker M, Tang SN, etal. Vaccine uptake and SARS-CoV-2 antibody prevalence among 207,337 adults during May 2021 in England: REACT-2 study. [Preprint.] medRxiv 2021:2021.07.14.21260497. doi: 10.1101/2021.07.14.21260497

10 Wei J, Stoesser N, Matthews PC, etalCOVID-19 Infection Survey team. Antibody responses to SARS-CoV-2 vaccines in 45,965 adults from the general population of the United Kingdom. Nat Microbiol 2021;6:1140-9. doi: 10.1038/s41564-021-00947-3. pmid: 34290390

11 Kamar N, Abravanel F, Marion O, Couat C, Izopet J, Del Bello A. Three doses of an mRNA covid-19 vaccine in solid-organ transplant recipients. N Engl / Med 2021;385:661-2. doi: 10.1056/NEJMc2108861. pmid: 34161700

12 Carr EJ, Wu M, Harvey R, etalHaemodialysis COVID-19 consortiumCrick COVID Immunity Pipeline. Neutralising antibodies after COVID-19 vaccination in UK haemodialysis patients. Lancet 2021:S0140-6736(21)01854-7. doi: 10.1016/S0140-6736(21)01854-7. pmid: 34391504

13 Khoury DS, Cromer D, Reynaldi A, etal. Neutralizing antibody levels are highly predictive of immune protection from symptomatic SARS-CoV-2 infection. Nat Med 2021;27:1205-11. doi: 10.1038/s41591-021-01377-8. pmid: 34002089

14 Whitehead M, Taylor-Robinson D, Barr B. Poverty, health, and covid-19. BMJ2021;372:n376. doi: 10.1136/bmj.n376. pmid: 33579719

15 Public Health England. Atlas of variation in risk factors and healthcare for vision in England. Public Health England, 2021.

16 Gill D, Baker EH, Hitchings AW. We need clinical guidelines fit for a pandemic. BMJ 2021;373:n1093. doi: 10.1136/bmj.n1093. pmid: 33926903

17 National Institute for Health and Care Excellence. The NICE strategy 2021 to 2026. 202 https:/www.nice.org.uk/about/who-we-are/corporate-publications/the-nice-strategy-2021-to2026

18 Taylor PC, Adams AC, Hufford MM, de la Torre I, Winthrop K, Gottlieb RL. Neutralizing monoclonal antibodies for treatment of COVID-19. Nat Rev Immunol 2021;21:382-93. doi: 10.1038/s41577-021-00542-x. pmid: 33875867

19 National Institute for Health and Care Excellence. Covid-19 rapid guideline: managing covid-19. NG 191. NICE, 2021

This article is made freely available for use in accordance with BMJ's website terms and conditions for the duration of the covid-19 pandemic or until otherwise determined by BMJ. You may use, download and print the article for any lawful, non-commercial purpose (including text and data mining) provided that all copyright notices and trade marks are retained. 